\title{
ARD
}

Annals of the Rheumatic Diseases

Leader

\section{Haemopoietic stem and progenitor cells in the treatment of severe autoimmune diseases}

The physician who regularly manages patients with severe autoimmune and connective tissue diseases faces, from time to time, the dilemma of a patient in whom conventional treatment fails to control life or organ threatening disease. So called conventional therapy mostly includes high doses of corticosteroids and some immunosuppressive regimen, usually cyclophosphamide. ${ }^{1}$ The number and diversity of such regimens alone speaks for their limitations, and the result is that in 1995 we are still discussing the role of plasmapheresis in systemic lupus erythematosus (SLE), ${ }^{2}$ the best management of lupus in pregnancy, ${ }^{3}$ and the correct treatment of the antiphospholipid antibody syndrome. ${ }^{4}$ Clearly, strong evidence exists that intensive immunosuppression may help significantly, ${ }^{5}$ the limiting factor often being the danger of too profound an immunosuppression, with resulting complications.

From haematology and solid tumour oncology, a relatively new technique has become available which may tip the balance in a positive direction: so called autologous stem cell therapy. A more correct term is intensive myelo and immunosuppression with autologous haemopoietic precursor cell support or rescue, as the precursor cells themselves are not the primary therapeutic agent and the reinfused cells are a combination of true stem cells and committed progenitor cells.

A true haemopoietic stem cell (HSC) should be self renewing, capable of differentiating into all cell types (including lymphocytes), and able to reconstitute fully a lethally irradiated animal, for example a mouse. Such cells constitute $<1 \%$ of bone marrow cells, and carry the surface markers CD34 and Thy-1, but are negative for lineage surface markers (Lin). A unique marker for HSCs has not been defined, though proof of their existence in man is provided by the long term haemopoietic reconstitution seen after allogeneic bone marrow transplantation. A true HSC generates at least one identical daughter cell during division, and is therefore self replenishing. The CD34 antigen persists into early progenitors (figure) which, during division, produce offspring that are always more differentiated than their parent cells. Therefore, although these still primitive progenitor cells are able to provide early reconstitution, this might not be sustained. The total CD34 positive, Thy-1 positive and Lin negative precursor cell population is about $3 \%$ of bone marrow cells.

HSCs may be obtained from bone marrow, peripheral blood or cord blood. They increase in the peripheral blood after intensive chemotherapy, for example with cyclophosphamide. For convenience, these cells may be driven out of the marrow by treatment with haemopoietic growth factors such as granulocyte colony stimulating factor (G-CSF) or granulocyte macrophage colony stimulating factor (GM-CSF), and harvested from peripheral blood. The mechanism of this is not fully understood. The process is called priming (see Glossary for explanation of this and other terms), and the cells obtained may be cryopreserved and stored. The patient then undergoes intensive myelo and immune ablation, as in solid tumour or leukaemia treatment, known as conditioning, and the stored HSCs are given back in order to reconstitute the haemopoietic and immune systems. Simplistically, the process is similar to a stored blood transfusion given back to the donor after elective surgery.

The HSCs may also be manipulated ex vivo, either to select positively for CD34 cells, or to eliminate other cells such as lymphocytes or tumour cells (purging). In addition, certain combinations of cytokines and growth factors may expand ex vivo progenitor cells, though true HSCs are probably expanded only minimally. In general, the more amplification occurs, the more the HSCs are pushed forward toward differentiation, and the fewer self

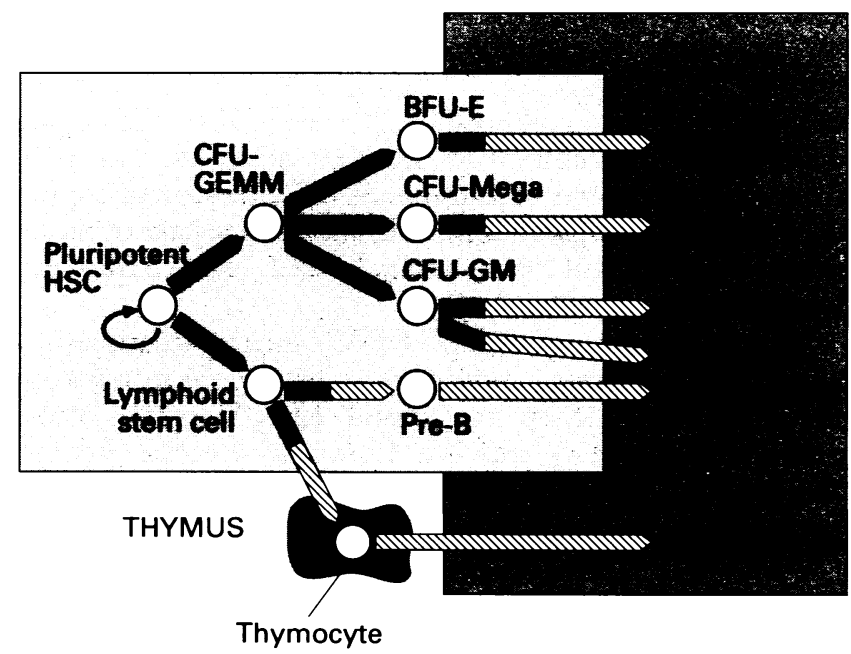

CD34 antigen expression in haemopoiesis. HSC = Haemopoietic stem cell; $C F U=$ colony forming unit; $G E M M=$ granulocytes, erythrocytes, monocytes, megakaryocytes; $B F U=$ burst forming unit; $E=$ erythrocyte; Mega = megakaryocyte; $G M=$ granulocyte macrophage; Pre- $B=B$ lymphocyte precursor. $\mathbf{D}=C D 34$ antigen expression; $\mathbb{Q}=C D 34$ negative. (Adapted after Auditore-Hargreaves K, et al. Bioconjugate Chem 1994; 5: 287-300.) 
replenishing cells remain. Preliminary data suggest that long term myeloreconstitution may be possible following such ex vivo manipulated stem cell support treatment. ${ }^{6}$

This process of myelo and immunoablation followed by autologous stem cell rescue carries a mortality (early, from infection before reconstitution is fully established, and from treatment related organ toxicity such as cardiac failure or interstitial pneumonitis) of around 3-5\%, and should not be confused with allogeneic bone marrow transplantation (BMT) with its intrinsic risk of graft versus host disease and overall greater mortality (15-35\%).

However, it was the observation in patients undergoing allogeneic BMT with coincident autoimmune disease (for example patients with rheumatoid arthritis (RA) who had gold or D-penicillamine induced aplastic anaemia), that the arthritis often remitted, that led to further consideration of BMT associated treatments. There are eight such cases of RA and aplasia documented: three of the patients died of transplant related mortality (with RA in remission); the remainder were alive at follow up ranging from two to eight years, ${ }^{7-10}$ with all but one in complete or partial $^{8}$ remission from RA. This last patient received a successful graft from her brother, but despite initial remission of her RA (clinically and serologically), it relapsed after two years. ${ }^{10}$ Similar remissions of $\mathrm{RA},{ }^{11}$ psoriasis, ${ }^{12}$ psoriatic arthritis, and ulcerative colitis ${ }^{13}$ have been recorded after allogeneic BMT for leukaemia. The converse observation has also been reported, namely passive transfer of autoimmune disease from the donor bone marrow through allogeneic BMT. Examples include myasthenia gravis, ${ }^{14}$ Graves disease, ${ }^{15}$ and autoimmune diabetes mellitus. ${ }^{16}$

A treatment programme based on autologous HSC rescue carries the risk that, if the basic defect of the autoimmune disease is in the stem cell, it will probably recur. If, however, the primary defect is an aberrent immune reaction to an acquired (for example viral) or self (for example cartilage) antigen, there is a theoretical chance that tolerance may be acquired in the newly reconstituted immune system-assuming ablation of memory cells.

As there are no published reports of such cases, one must examine the experimental animal data for suggestive clues. In a rat adjuvant arthritis model, Van Bekkum demonstrated cure with sublethal irradiation followed by histocompatible allogeneic BMT from a resistant strain and also, unexpectedly, with lethal irradiation followed by syngeneic or autologous $\mathrm{T}$ cell depleted BMT. ${ }^{17}$ Similar results have been obtained in rats for experimental allergic encephalomyelitis, ${ }^{18}$ and BMT is being discussed as a treatment for multiple sclerosis. ${ }^{19}$ In man, there are two reports of autologous HSC treatment coincidentally abrogating autoimmune disease: one case of myasthenia gravis and lymphoma, ${ }^{20}$ and one SLE and lymphoma. ${ }^{21}$

To consider the question of BMT in the treatment of autoimmune disease in humans, a liason group between the European Group for Blood and Marrow Transplantation (EBMT) and the European League Against Rheumatism (EULAR) has been formed and its preliminary recommendations were announced in Lancet, April $1995 .^{22}$ In essence, the European approach is one of a safety based open pilot study, with no irradiation, CD34 selection, or $\mathrm{T}$ cell depletion in the first step, unless strongly desired by the treating centre (which will include a BMT unit). Patients will be selected by two experienced physicians working in the field of rheumatology/clinical immunology who feel that conventional treatment has failed, that the situation is critical, and that significant reversible pathology exists. Only severe connective tissue cases will be considered in this early phase (SLE, scleroderma, dermatomyositis, polymyositis, and the vasculitides). RA will be considered at a later stage, when more safety data exist. The obvious aim in RA will be to select early those patients with a poor prognosis, such as those patients carrying the DR4 and DRw14 HLA types, ${ }^{23}$ and the 'shared RA epitope' of the HLA DRB1 allele, ${ }^{24}$ in order to avoid the late destructive stage with its associated morbidity and mortality.

Records of all patients treated in this way may be entered into the newly created EBMT compatible data bank prospectively, and after 12 months a decision to proceed with randomised comparative studies will be made according to the pilot study experience. This EBMT/ EULAR Stem Cell Project data base is available to any group wishing to enter patient details: participants will have continuous access to the data and modified Minimal Essential Data (MED A) forms are available. Similar activities are beginning in the USA and Australasia, with close collaboration and a shared desire for a common data base. Already, some selected patients, mostly with systemic sclerosis (scleroderma) and severe SLE, are being considered for autologous BMT in several centres in Europe and the USA.

It is likely that a step will be necessary to eliminate memory cells (for example, total body irradiation, $T$ cell depletion or radiomimetic drugs) if long lasting remissions are to be expected. In the Balb-c mouse model of experimental antiphospholipid syndrome, only $\mathrm{T}$ cell depleted syngeneic (pseudoautologous) BMT prevented recurrence of disease after total body irradiation. ${ }^{25}$ The problem associated with total body irradiation or radiomimetic drug treatment (for example with busulphan) is the risk of late malignancy. Because of this, and given the already large numbers of unknown factors in this area, the conditioning regimen now widely used in the treatment of aplastic anaemia (another autoimmune disease) has been recommended in the first instance. This will probably be modified as more experience and data are obtained. A recent study suggested that, in RA patients treated with oral cyclophosphamide, the increased risk of late tumour (relative risk 1.5) was mostly accounted for by skin and bladder tumours. ${ }^{26}$

During the many discussions leading up to the consensus statement, and subsequently, it became clear that haematologists and rheumatologists see the riskbenefit ratio very differently, because of the differing natural histories of the diseases we treat. At times wry, condescending smiles appeared on the EBMT faces as the rheumatologists discussed 'high dose' pulse cyclophosphamide treatment of lupus nephritis. Minutes later, the rheumatologists learned that $4 \mathrm{~g}$ of intravenous cyclophosphamide was part of a standard priming regimen, independent of subsequent immunoablation (conditioning). Each group needed to learn a new set of terminology (see Glossary) and the EULAR colleagues were impressed by the discipline and thoroughness of the EBMT approach to large multicentre trials. Guide and elder statesman of these discussions was Alberto Marmont, who for many years has discussed the issue and provided a bridge between the two groups. ${ }^{27}$

Only time, carefully performed trials, and data collection will show if the benefit outweighs the risk of this potentially powerful myelo and immunoablative treatment. An international meeting will be held on 27 and 28 November 1996 in Basel, under the auspices of the EBMT, EULAR and the Basel Institute for Immunology, to discuss stem cell biology, anecdotal experience so far obtained and, based on these data, the planning of prospective comparative trials. 
If the matter is correctly pursued, the hope is that in 20 years time there will be no further debate on the basic issue of BMT in autoimmune disease treatment, but rather either fine tuning of established protocols, or a faded memory of a proven failure.

University Department of Rheumatology,

Felix Platter Spital,

Burgfelderstrasse 101,

4055, Basel, Switzerland

University Department of Haematology, Kantonsspital Basel,

Petersgraben 4

4031, Basel, Switzerland

\section{Glossary}

Allogeneic-HLA matched individuals from the same species.

Autologous-Self; one only individual.

Syngeneic-Between individuals of a highly inbred strain or between identical twins.

Chimera-In allogeneic BMT, presence of donor type haemopoietic cells in the host. A 'complete chimera' is a successful graft.

Priming-Process of driving stem and progenitor cells out of the bone marrow for peripheral collection. Achieved with combinations or single use of G-CSF, GM-CSF, interleukin-3 (IL-3), cyclophosphamide (and other cytotoxic drugs).

Conditioning-Intensive immunosuppression to eliminate either tumour cells or putative pathogenic immunologically active cells. Implies suppression to an extent that a haemopoietic rescue is required.

Includes: Chemotherapy

Total body irradiation (TBI)

Total lymph node irradiation (TLI)

Serotherapy, for example:

Antilymphocyte globulin (ALG)

Monoclonal antibody (antithymocyte globulin)

Reconstitution-The development of a functioning bone marrow after engraftment. Implied also is the reconstitution of the immune system.

Purging-Ex vivo manipulation of the graft.

Either: Negative selection (killing unwanted cells with drugs or antibodies plus complement).

or: Positive selection (collecting and concentrating wanted cells with affinity columns; for example, stem cells with CD34 antibody on affinity column, fluorescence activated cell sorter, or long term cultures).

Sometimes the term purging is only used to imply negative selection. Ex vivo expansion - The use of various cocktails of growth factors and cytokines to expand wanted cell lines. Especially useful if limited numbers of valuable stem or progenitor cells are obtained through priming.

A typical combination is that recently published from Freiburg containing stem cell factor (SCF), IL-1, IL-3, IL-6, and erythropoietin in autologous serum.

Committed progenitor cells are expanded, but to date there is little evidence that true stem cells are significantly increased. Probably, the more expansion is attempted, the more cells are driven forward to commitment and the fewer uncommitted true stem cells remain. This may improve initial reconstitution, at the expense of later marrow exhaustion.

Stem cell-A cell capable of providing all mature cells in a particular lineage (for example haemopoietic and immune systems), and being capable of self replenishment (during division, one daughter cell temains pluripotent and the other becomes a progenitor).

Stem cells have been identified in many organs, including brain.

The haemopoietic stem cell is positive for the surface antigens CD34 and Thy-1, but negative for the lineage (Lin) markers. A similar pattern of surface markers is seen on primitive but committed progenitor cells. No unique stem cell marker has been identified. CD34 antigen-A $115 \mathrm{kDa}$ glycoprotein (at least three epitopes defined with monoclonal antibodies) with no sequence homology with other known molecules at either the protein or DNA level. Found on haemopoietic stem and early progenitor cells.

LTC-IC-Long Term Culture Inducing Cells. Probably represent true stem cells and very early progenitor cells.

Transplant product-The reinfused cells, with special reference to their source (bone marrow or peripheral blood) and type (autologous or allogeneic).
Recommended autologous transplant protocol for autoimmune diseases

Priming with cyclophosphamide $4 \mathrm{~g} / \mathrm{m}^{2}$, followed by G-CSF $10 \mu \mathrm{g} / \mathrm{kg}$ subcutaneously daily, or G-CSF alone if immediate immunosuppression is not indicated.

At least: $2 \times 10^{8}$ nucleated cells $/ \mathrm{kg}$ recipient body weight or $2 \times 10^{6} \mathrm{CD} 34$ cells $/ \mathrm{kg}$

or $2 \times 10^{4}$ granulocyte macrophage colony forming units $/ \mathrm{kg}$ should be harvested.

Conditioning with four doses of cyclophosphamide $50 \mathrm{mg} / \mathrm{kg}$ recipient body weight.

No recommendations concerning $\mathrm{T}$ cell depletion, positive CD34 cell selection, or total body irradiation are possible at this stage, therefore all such modifications should be recorded on the modified MED A forms (see text).

1 Fauci A S, Hayes B F, Katz P, et al. Wegeners granulomatosis: prospective clinical and therapeutic experience with 85 patients for 21 years. Ann Intern Med 1983; 98: 76-85.

2 Lewis E J. Plasmapheresis for lupus nephritis [Correspondence]. $N$ Engl f Med 1992; 14: 1029-30.

3 Lockshin M D. Treatment of lupus pregnancy: can we reach consensus? Clin Exp Rheum 1992; 10: 429-31.

4 Khamashta M A, Cuadrado M J, Mujic F, Taub N A, Hunt B J Hughes G R V. The management of thrombosis in the antiphospholipidantibody syndrome. N Engl F Med 1995; 332: 993-7.

5 Austin H A III, Klippel J H, Balow J E, et al. Therapy of lupus nephritis: Controlled trial of prednisone and cytotoxic drugs. N Engl f Med 1986; 314: 614-9.

6 Brugger W, Heimfeld S, Berenson R J, Mertelsmann R, Kanz L. Reconstitution of haemopoiesis after high dose chemotherapy by autologous progenitor cells generated ex-vivo. $N$ Engl f Med 1995; 333: 283-7.

7 Baldwin J L, Storb R, Donnall T E, Mannik M. Bone marrow transplantation in patients with gold-induced marrow aplasia. Arthritis Rheum 1977; 20: 1043-8.

8 Jacobs P, Vincent M D, Martell R W. Prolonged remission of severe refractory rheumatoid arthritis following allogeneic bone marrow transplantation for drug induced aplastic anaemia. Bone Marrow transplantation for drug
Transplant 1986; 1: 237-9.

9 Lowenthal R M, Cohen M L, Atkinson K, Biggs J C. Apparent cure of rheumatoid arthritis by bone marrow transplantation. $\mathcal{F}$ Rheumatol 1993; 20: $137-40$.

10 McKendry R J R, Huebsch L. Progression of rheumatoid arthritis following bone marrow transplantation (BMT). Arthritis Rheum 1994; 37 (suppl: ACR meeting): Abstract No 924

11 Roubenoff R, Jones R J, Karp J E, Stevens M B. Remission of rheumatoid arthritis with a successful treatment of acute myelogenous leukaemia with cytosine arabinoside, daunorubicin and M-AMSA. Arthritis Rheum 1987; 30: $1187-90$.

12 Eedy D J, Burrows D, Bridges J M, Jones F G. Clearance of severe psoriasis after allogeneic bone marrow transplantation. BMF 1990; 300: 908 .

13 Liu Yin J A, Jowitt S N. Resolution of immune-mediated diseases following allogeneic bone marrow transplantation for leukaemia. Bone Marrow Transplant 1992; 9: 31-3.

14 Grau J M, Casademont J, Monforte R, et al. Myasthenia gravis after allogeneic bone marrow transplantation: report of a new case and pathogenetic considerations. Bone Marrow Transplant 1990; 5: 435-7.

15 Aldouri M A, Ruggier R, Epstein O, Prentice H G. Adoptive transfer of hyperthyroidism and autoimmune thyroiditis following allogeneic bone marrow transplantation for chronic myeloid leukaemia. $\mathrm{Br} \mathcal{F}$ Haematol 1990; 74: 118-9.

16 Vialettes B, Maranchini D, San Marco M P, et al. Autoimmune polyendocrine failure-Type I (insulin-dependent) diabetes mellitus and polyendocrine failure-Type 1 (insulin-dependent) diabetes mellitus and patient with lymphoblastic leukaemia. Diabetologia 1993; 36: 541-6.

17 Knaan-Shanzer S, Houben P, Kinwell-Bohre E, Van Bekkum D W. Remission induction of adjuvant arthritis in rats by total body irradiation and autologous bone marrow transplantation. Bone Marrow Transplan 1991; 8: $10090-4$.

18 Van-Gelder M, Kinwell-Bohre E, Van Bekkum D W. Treatment of experimental allergic encephalomyelitis in rats by total body irradiation and syngeneic bone marrow transplantation. Bone Marrow Transplant 1993 11: $233-41$.

19 Burt R K, Burns W, Hess A. Bone marrow transplantation for multiple sclerosis. Bone Marrow Transplant 1995; 16: 1-6.

20 Salzman D, Tami J, Jackson C, et al. Clinical remission of myasthenia gravis (MG) in a patient (PT) after high dose therapy and autologous transplantation with CD34+ stem cells (SC). Blood 1994; 81 (suppl): 206a.

21 Fastenrath S, Dreger P, Schmitz N, Euler H. Autologous unpurged bone marrow transplantation in a patient with lymphoma and SLE: short term recurrence of antinuclear antibodies. Arthritis Rheum 1995; 38 (suppl) 303.

22 Marmont A M, Tyndall A, Gratwohl A, Vischer T. Haemopoietic precursorcell transplants for autoimmune diseases. Lancet 1995; 345: 978 .

23 Gough A, Faint J, Salmon M, et al. Genetic typing of patients with inflammatory arthritis at presentation can be used to predict outcome. Arthritis Rheum 1994; 37: 166-70.

24 Weyand C M, Hicok K C, Conn D L, Goronzy J J. The influence of HLA-DRB 1 gene on disease severity in rheumatoid arthritis. Ann Intern Med 1992; 117: 801-6.

25 Blank M, Krause I, Lanir N, et al. Transfer of experimental antiphospholipid syndrome by bone marrow transplantation. Arthritis Rheum 1995; 38: syndrome

26 Radis C D, Kahl L E, Baker G L, et al. Effects of cyclophosphamide on the development of malignancy and on long-term survival of patients with rheumatoic

27 Marmont A M. Autoimmunity and allogeneic bone marrow transplantation Bone Marrow Transplant 1992; 9: 1-3. 